\title{
A rare case of pituitary oncocytoma successfully treated with single-fraction stereotactic Gamma Knife surgery
}
Constantin Tuleasca ${ }^{1.3 .45}$, MD-PhD, Roy Thomas Daniel ${ }^{1.2}$, MBBS, MCh, Marc Levivier ${ }^{1,2}$, MD, $\mathrm{PhD}$, IFAANS

\begin{abstract}
${ }^{1}$ Lausanne University Hospital, Neurosurgery Service and Gamma Knife Center, ${ }^{2}$ University of Lausanne (Unil), Faculty of Biology and Medicine (FBM), ${ }^{3}$ Ecole Polytechnique Fédérale de Lausanne (EPFL), Lausanne, Switzerland; ${ }^{4}$ Sorbonne Université, Faculté de Médecine, ${ }^{5}$ Assistance Publique-Hôpitaux de Paris, Hôpitaux Universitaires Paris-Sud, Centre Hospitalier Universitaire Bicêtre, Paris, France;
\end{abstract}

Running title: Radiosurgery for oncocytoma

Key words: pituitary, oncocytoma, radiosurgery, Gamma Knife

Funding: Constantin Tuleasca gratefully acknowledges receipt of a 'Young Researcher in Clinical Research Grant' (Jeune Chercheur en Recherche Clinique) from the University of Lausanne (UNIL), Faculty of Biology and Medicine (FBM) and the Lausanne University Hospital (CHUV)

Acknowledgments: Lausanne University Hospital and University of Lausanne.

Conflict of interest: No conflict of interest.

\section{Corresponding author:}

Constantin Tuleasca, MD-PhD, Assistance Publique-Hôpitaux de Paris, Hôpitaux Universitaires ParisSud, Centre Hospitalier Universitaire Bicêtre, Service de Neurochirurgie, Paris, France; Sorbonne Université, Faculté de Médecine; Centre Hospitalier Universitaire Vaudois, Neurosurgery Service and Gamma Knife Center, Rue du Bugnon 44-46, BH-08, CH-1011, Lausanne, Switzerland; Tel: +41-21-314-26-02; Fax: +41-21-314-11-99; e-mail: constantin.tuleasca@gmail.com ORCID number : 0000-0001-6776-1486 


\section{Dear Editor,}

We read with great interest the article of Oushy et al.[1] evaluating the largest cohort of extremely rare pituitary spindle cell oncocytoma (SCO) treated by single-fraction stereotactic radiosurgery (SRS). We agree with the author's conclusion that these uncommon sellar lesions have a propensity for progression or recurrence. Adjuvant SRS after subtotal resection or at the time of recurrence can be a viable alternative.

In our SRS practice in Lausanne, during the past nine years, we also treated one case of SCO, which benefitted from initial gross subtotal resection by transsphenoidal approach approximately 5 and half years prior to SRS (Figure 1, A). The anatomopathological result revealed a MIB1 between 3-5\%. The residual SCO had been visualized on 16 months followup MRI (Figure 1, B) and had already displayed slight tumor progression at 40 months after microsurgery (Figure 1,C), yet remaining at distance from the optic apparatus. At 64 months after microsurgery (Figure 1, D) there was further SCO progression, approaching the optic pathways, which were now located closer from the tumor, still allowing safe SRS treatment by Gamma Knife due to its steep gradient[2] (GK, Figure 1, D). By analogy to non-functional pituitary adenomas, we (CT, ML) prescribed a marginal dose of 16 Gy at the $50 \%$ isodose line. The target volume (TV) was $0.352 \mathrm{ml}$ and the prescription isodose volume was $0.572 \mathrm{ml}$ (maximal dose received by optic pathways 4.3 Gy). Follow-up MRI displayed dramatic volumetric decreased at 6 months and further at 1 year, which persisted up to 3.5 years after RS (Figure 1, E).

We congratulate the authors for fulfilling a gap of knowledge in this rare indication[1]. Our case adds to the sparse literature on this pathology, while showing also the efficacy of SRS by single-fraction GK in this indication. The median marginal dose used by the authors was 17 Gy (range 14-20), being safe and effective. Careful attention should be paid to the dose received by the optic apparatus[3], due to its proximity in this indication. Furthermore, one should also consider the risk of new or worsened panhypopituitarism, after single fraction SRS, which might be as high as $21 \%$ according to some studies [4]. In this context, reducing radiation exposure to the identifiable gland to a mean dose $<11.0$ Gy whenever possible may lower the incidence of new hormonal deficits after pituitary adenoma SRS [5]. 
Figure 1: A- MRI in coronal, axial and sagittal plane, before microsurgery; B- MRI at 16 months after microsurgery, showing residual spindle cell oncocytoma (SCO, arrow) and C- 40 months after microsurgery, showing slight progression of the residual SCO (arrow); D- MRI at 64 months after microsurgery, with Gamma Knife radiosurgery planning; the dosimetry is colored in yellow, while the optic apparatus in magenta; the 8 Gy isodose line corresponding to the optic pathways is displayed in green; E- three years and a half follow-up MRI, showing marked volumetric decrease

\section{References}

1. Oushy, S., et al., Single-fraction stereotactic radiosurgery for spindle cell oncocytoma: preliminary experience and systematic review of the literature. J Neurooncol, 2019.

2. Tuleasca, C., et al., Gamma Knife radiosurgery for cervical spine lesions: expanding the indications in the new era of Icon. Acta Neurochir (Wien), 2016. 158(11): p. 2235-2236.

3. Leavitt, J.A., et al., Long-term evaluation of radiation-induced optic neuropathy after single-fraction stereotactic radiosurgery. Int J Radiat Oncol Biol Phys, 2013. 87(3): p. 524-7.

4. $\quad$ Sheehan, J.P., et al., Gamma Knife radiosurgery for the management of nonfunctioning pituitary adenomas: a multicenter study. J Neurosurg, 2013. 119(2): p. 446-56.

5. Graffeo, C.S., et al., Hypopituitarism After Single-Fraction Pituitary Adenoma Radiosurgery: Dosimetric Analysis Based on Patients Treated Using Contemporary Techniques. Int J Radiat Oncol Biol Phys, 2018. 101(3): p. 618-623. 

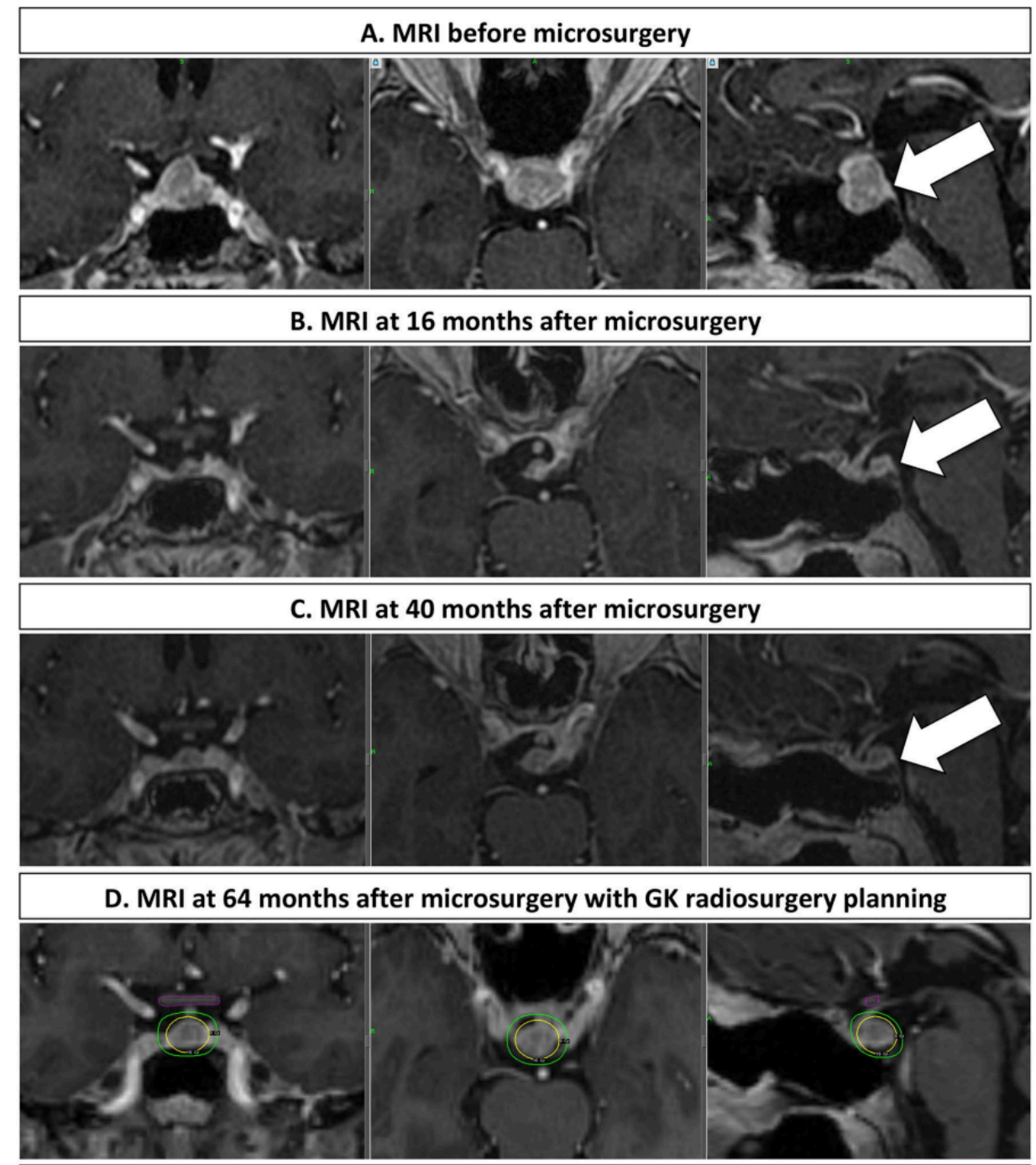

E. MRI at 3.5 years after GK radiosurgery

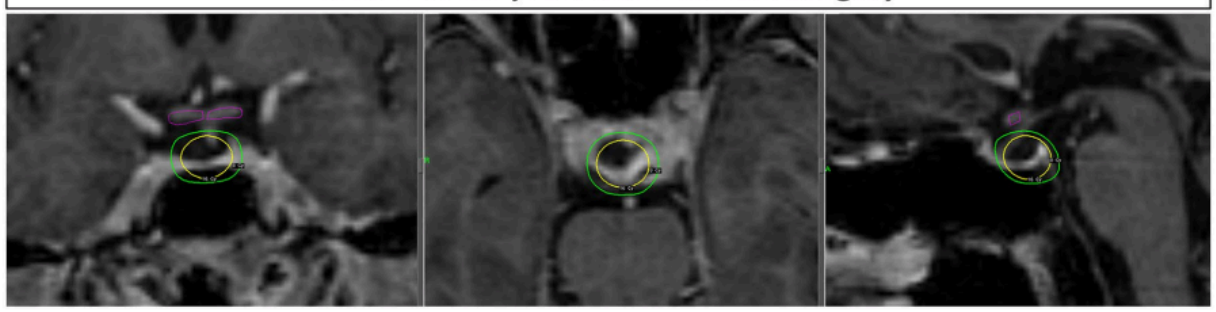

